Rev. Int. Contam. Ambie. 37, 109-117, 2021

https://doi.org/10.20937/RICA.53701

\title{
EFICACIA DEL PERMANGANATO POTÁSICO EN LA DEGRADACIÓN DE CIANURO Y TIOCIANATO EN AGUAS RESIDUALES MINERAS
}

\author{
E ffectiveness of potassium permanganate for the degradation of cyanide and thiocyanate from mine wastewater
}

\author{
Begoña FERNÁNDEZ PÉREZ ${ }^{1 *}$, Juan José VIÑA MEDIAVILLA ${ }^{1}$, \\ Julia AYALA ESPINA ${ }^{1}$ y Conchi O. ANIA ${ }^{2,3}$
}

${ }^{1}$ Escuela Técnica Superior de Ingeniería de Minas, Universidad de Oviedo, 33001 Oviedo, España.

${ }^{2}$ CEMHTI, CNRS (UPR 3079), Université d'Orléans, 45071 Orléans, France.

${ }^{3}$ Instituto Nacional del Carbón (INCAR-CSIC), Apdo. 73, 33080 Oviedo, España.

*Autora para correspondencia: fernandezbegona@uniovi.es

(Recibido: agosto 2019; aceptado: abril 2020)

Palabras clave: especies cianuradas, oxidación química, aguas ácidas de mina.

\begin{abstract}
RESUMEN
El objetivo de este trabajo es evaluar la viabilidad del uso de permanganato de potasio como método eficaz para la degradación de aguas residuales procedentes del sector de la minería aurífera que contienen cianuros y tiocianatos. Para ello se llevó a cabo la degradación simultánea de ambas especies cianuradas en aguas industriales. Los resultados indican que el permanganato de potasio es un agente oxidante eficaz para la eliminación de cianuro de aguas residuales de la minería aurífera. Se lograron porcentajes de eliminación de cianuro entre 95 y $100 \%$, en aguas con una concentración inicial de cianuros de $312 \mathrm{mg} / \mathrm{L}$ y de tiocianatos de $314 \mathrm{mg} / \mathrm{L}$. En cambio, la eficiencia de degradación de tiocianatos es mucho menor, con porcentajes de eliminación inferiores al $50 \%$ para altas concentraciones de oxidante. Los estudios oxidativos en aguas sintéticas mostraron que la oxidación parcial de tiocianatos da lugar a la liberación de cianuros en el agua. Por este motivo, la oxidación simultánea de cianuros y tiocianatos en las aguas residuales estudiadas no es completa, encontrándose concentraciones residuales, no despreciables, de ambas especies cianuradas, tras la reacción con el permanganato. Esto hace necesario aplicar otros métodos de oxidación para la eliminación total de las especies cianuradas.
\end{abstract}

Key words: cyanide species, chemical oxidation, acid mine wastewater.

\begin{abstract}
This work was focused on evaluating the viability of potassium permanganate as an oxidizing agent for the simultaneous degradation of cyanides and thiocyanates from an acid mine wastewater. Data has confirmed the effectiveness of permanganate solutions for the oxidation of cyanides, with removal efficiencies between 95-100\%, in waters with an initial concentration of cyanides of $312 \mathrm{mg} / \mathrm{L}$ and thiocyanates of $314 \mathrm{mg} / \mathrm{L}$. In contrast, the chemical oxidation of thiocyanates with permanganate was less efficient, with overall yields below $50 \%$ for the highest oxidant concentrations assayed. Furthermore, the studies from synthetic water confirmed that the partial oxida-
\end{abstract}


tion of thiocyanates using permanganate results in the release of a residual amount of cyanides that remains in the treated water. For this reason, the simultaneous oxidation of cyanides and thiocyanates in not complete for any of the studied industrial wastewaters, finding not negligible residual concentrations of both cyanide and thiocyanate, after the reaction with permanganate. This makes it necessary to consider further treatments of the wastewater for a full removal of the cyanide species.

\section{INTRODUCCIÓN}

El cianuro y el tiocianato son dos compuestos tóxicos que se encuentran frecuentemente en las aguas residuales del procesamiento de minerales de oro y de la fabricación de coque para la producción de acero (aleación de hierro) como componente mayoritario, y otros elementos como carbono, manganeso, fósforo, níquel, azufre, cromo, etc. (Bonan et al. 1994, Logsdon et al. 1999, Vicente y Díaz 2003). El tiocianato, que también se forma biológicamente a partir de la degradación del cianuro, es menos tóxico, pero más estable que este último; por tanto, es más difícil de degradar (Douglas et al. 2012).

Las reglamentaciones ambientales de un gran número de países limitan los niveles de cianuro en agua. Por ejemplo, la Organización Mundial de la Salud fija un valor inferior a $0.07 \mathrm{mg} / \mathrm{L}$ en agua potable (OMS 2003). Países como México han adoptado esta recomendación (SSA 1994), mientras que Canadá, Estados Unidos, Chile, Argentina y Brasil, entre otros, permiten concentraciones mayores de hasta $0.2 \mathrm{mg} / \mathrm{L}$ (OMS 2003). No obstante, no existe legislación directa acerca de la liberación de tiocianato en el ambiente, aunque varias agencias reguladoras han publicado directrices de vigilancia de su concentración en agua (Dash et al. 2009) con base en estudios sobre la toxicidad directa o indirecta de ambos compuestos en vertebrados (principalmente problemas respiratorios y mortalidad).

Los altos precios del oro a principio del presente siglo dieron como resultado un aumento en la producción de este metal, haciendo rentables aquellas minas que habían dejado de serlo por el aumento de inversiones en explotación y desarrollo de nuevas técnicas de extracción (Brady 2017). El método más común de extracción del oro en la industria es la lixiviación con cianuro (Ballester et al. 2003). De acuerdo con la literatura, las concentraciones de cianuros y tiocianatos en aguas de proceso generadas en concentradores de mineral de oro pueden variar entre $40 \mathrm{mg} / \mathrm{L}$ y $700 \mathrm{mg} / \mathrm{L}$ (Soto et al. 1995, Logsdom et al. 2004), lo que hace necesaria su eliminación antes del vertido final del efluente generado al ambiente.
Se han estudiado diferentes técnicas de degradación para el tratamiento de corrientes contaminadas con cianuro y tiocianato (Kuyucaka y Akcil 2013, Botz et al. 2016). Los tratamientos naturales, biológicos u oxidativos son los más ampliamente utilizados (Dabrowski et al. 2002, Budaev et al. 2014). En el caso de los tiocianatos, menos estudiados, el tratamiento biológico resulta, en algunos casos, una solución simple y la opción más económica (Medina et al. 2012, Rancaño 2012). Sin embargo, la aplicación del tratamiento biológico se limita a bajas concentraciones de tiocianato. Por otra parte, la presencia de ambas especies cianuradas con frecuencia inhibe la actividad de las bacterias, disminuyendo por tanto la eficiencia de la degradación (Douglas et al. 2012, Mekuto et al. 2017).

La degradación de cianuros ha sido más profusamente estudiada y se han propuesto un gran número de métodos de eliminación (Botz et al. 2016). Entre los más utilizados destacan los oxidativos, siendo los más empleados en plantas mineras en la actualidad el proceso Degüssa, que utiliza peróxido de hidrógeno como oxidante (Botz et al. 2005), y el proceso INCO (International Nickel Company), que utiliza una mezcla de $\mathrm{SO}_{2}$ /aire como oxidante (Devuyst et al. 1982). No obstante, ambos métodos presentan el inconveniente de un alto consumo de reactivos debido a que los oxidantes también reaccionan con otros compuestos presentes en las aguas residuales. Por ello es habitual que se realicen varios ciclos de tratamiento de las aguas residuales con el mismo proceso, o bien tratamientos sucesivos empleando varios métodos de degradación, para lograr los límites requeridos por la legislación ambiental vigente. Asimismo, es habitual que las aguas de proceso (con concentraciones de especies cianuradas superiores a las permitidas) se almacenen en estanques de relave donde se produce la degradación natural de contaminantes antes de su vertido. Este tipo de práctica entraña un riesgo ambiental alto debido a posibles fallos en las instalaciones de contención durante el almacenamiento.

Es poco probable que las preocupaciones sociales y ambientales sobre la minería que utiliza cianuro 
para la extracción de oro de sus menas se disipen en un futuro cercano. La combinación de imágenes negativas inherentes al uso del cianuro, así como los problemas ambientales asociados con este tipo de minería, hace que este proceso continúe generando críticas, por lo que es necesario buscar un método que permita la eliminación efectiva de ambos contaminantes.

En este sentido, el permanganato de potasio $\left(\mathrm{KMnO}_{4}\right)$ es un oxidante fuerte (Medialdea et al. 2005), ampliamente utilizado para el tratamiento y desinfección del agua (Marecaux 2006). Se usó por primera vez en 1910 para el tratamiento del agua potable en Londres (Pizzi 2010). En la actualidad se usa comúnmente en la industria para la seguridad de la salud ambiental (Degrémont 1991).

Estudios previos han demostrado que el $\mathrm{KMnO}_{4}$ se comporta como un agente de degradación efectivo del cianuro (Fernández 2007, Sancho et al. 2009, Ordiales et al. 2015), con excelentes resultados en aguas residuales sintéticas y naturales de la extracción de oro.

Para lograr una reacción de oxidación segura y efectiva, ésta debe llevarse a cabo en condiciones fuertemente alcalinas ( $\mathrm{pH} 12-14$; e.g., el potencial redox del par $\mathrm{MnO}_{4}^{-} / \mathrm{Mn}^{2+}$ en medio básico es de $+0.82 \mathrm{~V}$ ). En estas condiciones, el $\mathrm{KMnO}_{4}$ puede transformar el cianuro en cianato y otros derivados con menos toxicidad (Vella 1996, Fernández 2007).

El objetivo de este trabajo es evaluar la viabilidad y eficacia de la utilización de $\mathrm{KMnO}_{4}$ para el tratamiento de aguas residuales del proceso de extracción de oro con altas concentraciones de cianuro $\mathrm{y}$ tiocianato.

\section{MATERIALES Y MÉTODOS}

\section{Aguas residuales mineras}

Se estudiaron cuatro muestras de aguas residuales con concentraciones diferentes, procedentes de una instalación minera local que emplea el proceso de cianuración utilizando carbón en la lixiviacion (CIL, por sus siglas en inglés) para la disolución del oro (Elsner 1846) y el proceso INCO para la degradación de las especies cianuradas del efluente acuoso generado (Yarar 2002). Las muestras de agua utilizadas en este trabajo proceden de cuatro ubicaciones diferentes de dicha instalación: $i$ ) agua del tanque de tratamiento de cianuración (CIL); ii) agua de proceso (AP), procedente de la planta de tratamiento metalúrgico de la instalación; iii) aguas de proceso pretratadas (APT) procedentes de la degradación de las aguas del tanque CIL con el proceso INCO, y $i v$ ) aguas de drenaje $(\mathrm{AD})$ de la balsa de contención. Esta selección de muestras de agua de distintas corrientes permitió evaluar la eficacia del método en un amplio abanico de concentraciones de cianuro, con valores de 312, 40-50, 15 y $1.9 \mathrm{mg} / \mathrm{L}$ de cianuro para las aguas CIL, AP, APT y AD, respectivamente. La concentración de tiocianatos de las distintas aguas se encuentra en valores de 300 a $600 \mathrm{mg} / \mathrm{L}$. Las aguas poseen además un $\mathrm{pH}$ de 8 , valores de conductividad de $1050 \mu \mathrm{S} / \mathrm{cm}$, sólidos en suspensión en $49 \mathrm{mg} / \mathrm{L}$ y demanda química de oxígeno de $333 \mathrm{mg} / \mathrm{L}$. El análisis de los metales comunes de estas aguas arrojó los siguientes valores medios (mg/L): $\mathrm{Zn}: 0,10$; Cd: 0,04; Co: 0,20; Na: 600; K: 26; Ca: 65; Cu: 80. El alto contenido de cobre se debe a la mineralogía de la mena. $\mathrm{El} \mathrm{Cu}$ es un catalizador de la reacción de oxidación del cianuro, ayudando además a la floculación y posterior decantación del depósito formado (Vella 1996). Otros elementos, como $\mathrm{Sn}, \mathrm{Cr}$ o Pb, se detectaron en concentraciones inferiores a $5 \mu \mathrm{g} / \mathrm{L}$.

\section{Aguas sintéticas}

Con objeto comparativo, se utilizaron aguas sintéticas de cianuros y tiocianatos con concentraciones de $100,200,400$ y $500 \mathrm{mg} / \mathrm{L}$ para los cianuros, y de 50 a $200 \mathrm{mg} / \mathrm{L}$ para los tiocianatos. Las aguas se prepararon disolviendo cianuro y tiocianato potásico en agua destilada. El pH resultante de estas disoluciones fue de 8 .

\section{Procedimiento de oxidación}

Los ensayos de oxidación con permanganato potásico se realizaron en condiciones básicas en todos los casos, fijando el $\mathrm{pH}$ por encima de 12 mediante la adición de óxido de calcio. Tras alcanzar la basicidad requerida en el agua sin tratar, se puso en contacto el agua residual con la cantidad necesaria de permanganato potásico para cada determinada relación másica. Para minimizar cambios volumétricos por dilución durante la adición de reactivos, se empleó una de disolución madre de permanganato potásico de elevada concentración (e.g., $2.5 \%$ en peso). La disolución resultante se mantuvo en agitación durante 20 min (Fernández 2007). Las muestras se filtraron para eliminar los lodos generados, y seguidamente se analizó la concentración de especies cianuradas remanentes en disolución. Con objeto de optimizar la cantidad de oxidante necesaria para llevar a cabo la oxidación de ambas especies, se utilizaron relaciones másicas $\mathrm{KMnO}_{4}: \mathrm{CN}$ variables de 2 a 7 , y relaciones másicas $\mathrm{KMnO}_{4}: \mathrm{SCN}$ variables de 1 a 7 . Los ensayos se realizaron en tanques de $4 \mathrm{~L}$ de capacidad. 
Con objeto de corroborar las condiciones óptimas de tratamiento establecidas previamente en la bibliografía para la aplicación de este método oxidativo, se realizaron además ensayos con aguas sintéticas con distintas concentraciones de cianuro de potasio en solución empleando un procedimiento similar.

\section{Determinación de concentraciones de cianuros y tiocianato}

La concentración de cianuros en aguas reales se determinó mediante el método del ácido pícrico (Fernández 2007), ya que es el método analítico empleado en la instalación minera local. Su fundamento es la formación de un complejo coloreado entre el cianuro y el ácido pícrico en presencia de níquel, cuya concentración se detecta fácilmente por espectrofotometría visible ultravioleta, fijando la longitud de onda en $520 \mathrm{~nm}$.

Paralelamente se utilizó un método de análisis de inyección en flujo para la determinación de cianuros totales en las aguas sintéticas, según la metodología de la norma UNE-EN ISO 14403-1:2012 (UNE 2012). Este método se basa en la detección colorimétrica a $610 \mathrm{~nm}$ del compuesto generado por reacción de los cianuros en presencia de cloramina $\mathrm{T}$, ácido isonicotínico, y 1,3 ácido dimetil barbitúrico (Nagashima y Ozawa 1981). El tiocianato no interfiere con esta determinación.

Finalmente, las concentraciones de tiocianato y de los intermedios de oxidación se detectaron por medio de un cromatógrafo iónico (850 Professional IC, Metrohm), provisto de detectores de conductividad y sendas columnas específicas para la determinación de aniones (Metrosep A Supp 7-250, $4 \mathrm{~mm} \times 250 \mathrm{~mm}, 5$ $\mu \mathrm{m}$ ), y cationes (Metrosep C3 250/4.0, $4 \mathrm{~mm} \times 250$ $\mathrm{mm}, 5 \mu \mathrm{m})$, siguiendo las pautas establecidas en la norma UNE-EN ISO 10304-1:2009 (UNE 2009). Las columnas se mantuvieron a $40{ }^{\circ} \mathrm{C}$, y como fase móvil se emplearon $0.5 \mathrm{~mL} / \mathrm{min}$ de $3.5 \mathrm{mM} \mathrm{HNO}_{3}$ para la detección de cationes y $3.6 \mathrm{mM} \mathrm{Na}_{2} \mathrm{CO}_{3}$ para la detección de aniones. El volumen de inyección para todas las muestras fue de $20 \mu \mathrm{L}$. Todas las muestras se filtraron con un filtro de nylon de $0.45 \mu \mathrm{m}$ antes de realizar los análisis.

\section{RESULTADOS Y DISCUSIÓN}

\section{Aguas sintéticas}

La figura 1a presenta el porcentaje de eliminación de cianuros para diferentes relaciones másicas $\mathrm{KMnO}_{4}: \mathrm{CN}$ en una disolución de $200 \mathrm{mg} / \mathrm{L}$ de concentración inicial. Como se puede apreciar, al aumentar la cantidad de permanganato aumenta la degradación de cianuros, alcanzándose únicamente la eliminación completa para la relación $\mathrm{KMnO} 4: \mathrm{CN}$ de 7. Esto se debe a que, salvo en este último caso, se trabaja en condiciones de defecto de oxidante, con consumos de permanganato del $100 \%$. En cambio, el consumo de permanganato para la relación $\mathrm{KMnO} 4: \mathrm{CN}$ de 7 fue del $75 \%$, lo que confirma que es necesario optimizar la dosis de permanganato para alcanzar la degradación completa.
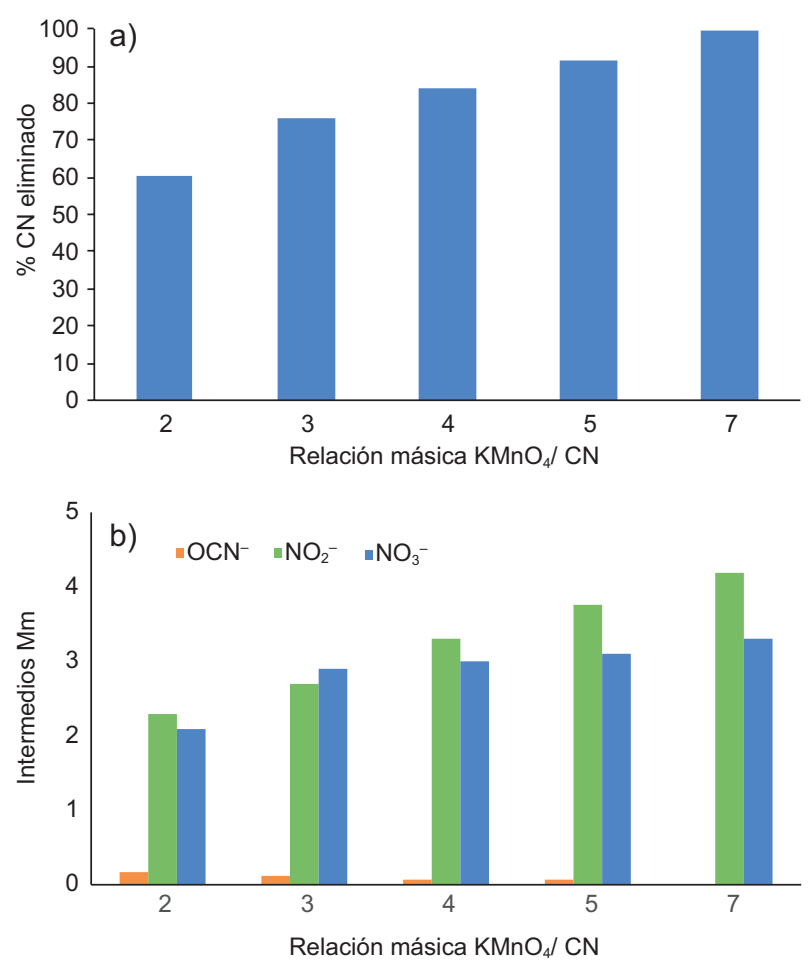

Fig. 1. (a) Eliminación de cianuro $(\mathrm{CN})$ a partir de agua sintética de $200 \mathrm{mg} / \mathrm{L}$ de concentración inicial para diferentes relaciones másicas $\mathrm{KMNO}_{4} / \mathrm{CN}$. (b) Concentración de intermedios de oxidación detectados en disolución.

La figura 1b muestra la distribución de intermedios de oxidación. Tal como se puede apreciar, no se detectaron cianatos para la relación $\mathrm{KMnO} 4: \mathrm{CN}$ de 7. Sin embargo, con relaciones másicas inferiores aparecen (pero en pequeña cantidad) lo que está de acuerdo con la literatura, ya que la oxidación de cianuros transcurre a través de la formación de cianatos según las reacciones 1-3 (Oulego et al. 2014):

$$
\begin{aligned}
& 2 \mathrm{Mn}_{4}^{-}+3 \mathrm{CN}^{-}+\mathrm{H}_{2} \mathrm{O} \rightarrow \\
& 2 \mathrm{MnO}_{2}+3 \mathrm{CNO}^{-}+2 \mathrm{OH}^{-} \\
& 2 \mathrm{CNO}^{-}+4 \mathrm{OH}^{-} \rightarrow 2 \mathrm{CO}_{2}+\mathrm{N}_{2}+2 \mathrm{H}_{2} \mathrm{O}
\end{aligned}
$$


$2 \mathrm{CNO}^{-}+2 \mathrm{H}_{2} \mathrm{O} \rightarrow \mathrm{CO}_{3}^{2-}+\mathrm{NH}_{4}^{+}$

$2 \mathrm{NH}_{4}^{+}+3 \mathrm{O}_{2} \rightarrow 2 \mathrm{NO}_{2}^{-}+4 \mathrm{H}^{+}+2 \mathrm{H}_{2} \mathrm{O}$

$2 \mathrm{NO}_{2}^{-}+\mathrm{O}_{2} \rightarrow 2 \mathrm{NO}_{3}^{-}$

Esto indica que la hidrólisis de este compuesto para generar amonio $u$ otros intermedios (reacciones 2,3 ) es muy rápida, tal como ha sido reportado para otros sistemas de oxidación (Oulego et al. 2014). No obstante, se detectaron nitritos y nitratos, lo que sugiere la oxidación de iones amonio generados tras la hidrólisis de cianato (reacciones 4,5). La presencia de permanganato y oxígeno disuelto favorecerían estas reacciones.

En el cuadro I se muestran los resultados obtenidos para distintos ensayos utilizando la relación másica de 5:1 de KMnO4:CN y variando la concentración de cianuros desde 100 hasta $500 \mathrm{mg} / \mathrm{L}$, así como los porcentajes de degradación de cianuros y la concentración de intermedios generados durante el proceso oxidativo. Los resultados indican que la degradación de cianuros aumenta a medida que desciende la concentración inicial de cianuros. Esto se debe a que, para estas relaciones másicas y concentraciones de cianuros, el permanganato potásico es el reactivo limitante de la oxidación, puesto que se consume completamente para todas las condiciones (con excepción de la disolución de $100 \mathrm{mg} / \mathrm{L}$ de concentración inicial, para la cual se obtuvo un porcentaje de oxidación del $99 \%$ ).

Es interesante destacar que el balance molar de cianuro degradado respecto a los intermedios detectados en disolución (cuadro I) es cercano a 1 en todos los casos. Esto sugiere que no han producido pérdidas por volatilización de especies gaseosas, tales como nitrógeno y amoniaco, durante la reacción.

En la figura 2a se presentan los resultados de los ensayos realizados con aguas sintéticas con $200 \mathrm{mg} / \mathrm{L}$ de tiocianato potásico y diferentes relaciones másicas de $\mathrm{KMnO}_{4}: \mathrm{SCN}$. Se puede observar que el porcentaje de eliminación de tiocianatos aumenta con la cantidad de permanganato empleada; no obstante, el porcentaje máximo de eliminación para las condiciones estudiadas no supera el $30 \%$, incluso para las relaciones másicas más elevadas. Esto contrasta con los resultados obtenidos para la degradación de cianuros (Fig. 1a) en condiciones similares, en la cual se alcanzaban eliminaciones superiores al $50 \%$ en todos los casos.
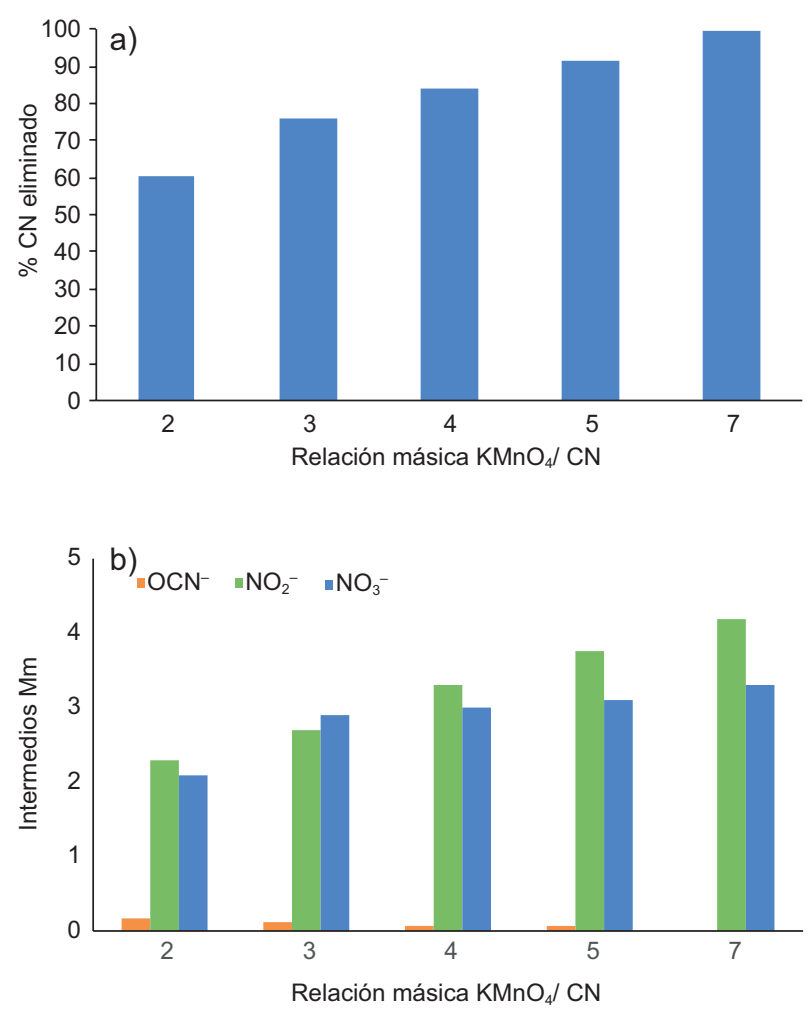

Fig. 2. (a) Eliminación de tiocianato $(\mathrm{SCN})$ a partir de agua sintética de $200 \mathrm{mg} / \mathrm{L}$ de concentración inicial para diferentes relaciones másicas $\mathrm{KMnO}_{4} / \mathrm{CN}$. (b) concentración de intermedios de oxidación detectados en disolución.

CUADRO I. ELIMINACIÓN DE CIANURO Y CONCENTRACIÓN DE ESPECIES INTERMEDIAS DETECTADAS PARA AGUAS SINTÉTICAS DE CIANURO*.

\begin{tabular}{cccccc}
\hline $\begin{array}{c}\text { KCN inicial } \\
(\mathrm{mg} / \mathrm{L})\end{array}$ & $\begin{array}{c}\mathrm{KCN} \text { inicial } \\
(\mathrm{mM})\end{array}$ & $\begin{array}{c}\mathrm{CN} \text { degradado } \\
(\%)\end{array}$ & $\begin{array}{c}\mathrm{NO}_{2} \\
(\mathrm{mM})\end{array}$ & $\begin{array}{c}\mathrm{NO}_{3}{ }^{-} \\
(\mathrm{mM})\end{array}$ & $\begin{array}{c}\text { Relación molar } \\
\text { intermedios/CN }\end{array}$ \\
\hline 100 & 3.9 & 99 & 2.4 & 1.4 & 0.99 \\
200 & 7.7 & 91 & 3.8 & 3.1 & 0.98 \\
400 & 15.4 & 86 & 8.5 & 4.3 & 0.96 \\
100 & 19.2 & 77 & 9.2 & 5.4 & 0.99 \\
\hline
\end{tabular}

*Concentración variable y relación másica $\mathrm{KMnO}_{4} / \mathrm{CN}$ de 5:1; pH = 12 y 20 min de agitación. 
En cuanto a los intermedios de oxidación, se detectaron cantidades apreciables de cianato, cianuro, nitrato, nitrito, amonio y sulfato (Fig. 2b). La presencia de cianuros como intermedio de degradación de tiocianatos en disoluciones sintéticas indica que el mecanismo de oxidación procede a través de la siguiente reacción:

$6 \mathrm{Mn}_{4}^{-}+\mathrm{SCN}^{-}+8 \mathrm{OH}^{-} \rightarrow$

$6 \mathrm{MnO}_{3}^{2-}+\mathrm{SO}_{4}^{2-}+\mathrm{CN}^{-}+4 \mathrm{H}_{2} \mathrm{O}$

seguida de las reacciones anteriormente señaladas para la degradación de cianuro.

Por otro lado, la presencia de sulfatos confirma la oxidación de la molécula de tiocianato por acción del permanganato como oxidante (a pesar de los bajos porcentajes de eliminación obtenidos). Un mecanismo similar ha sido propuesto para la oxidación de tiocianato a partir de otros oxidantes (ozono, peróxido de hidrógeno, fotoquímica) (Chung y Wood 1970, Collado et al. 2009, 2010, Douglas et al. 2012, Viña et al. 2019). Asimismo, es interesante destacar que en el caso de la oxidación de tiocianato, al contrario de lo que se observó para el cianuro, es posible detectar cianato antes de su hidrólisis.

En el cuadro II se recogen los intermedios generados durante la oxidación de las aguas sintéticas de tiocianato con concentraciones iniciales entre $50 \mathrm{y}$ $200 \mathrm{mg} / \mathrm{L}$, para una relación másica $\mathrm{KMnO}_{4}: \mathrm{SCN}$ de $3: 1$. Se debe destacar que en todas las concentraciones iniciales la cantidad de nitratos y nitritos es superior a la concentración de amonio detectada. Asimismo, se detectó una concentración mayor de intermedios

CUADRO II. ELIMINACIÓN DE TIOCIANATO Y CONCENTRACIÓN DE ESPECIES INTERMEDIAS DETECTADAS PARA AGUAS SINTÉTICAS DE TIOCIANATO CONCENTRACIÓN VARIABLE*

\begin{tabular}{lccc}
\hline & $200 \mathrm{mg} / \mathrm{L}$ & $100 \mathrm{mg} / \mathrm{L}$ & $50 \mathrm{mg} / \mathrm{L}$ \\
\hline KSCN inicial (mM) & 3.45 & 1.72 & 0.86 \\
SCN degradado (\%) & 19 & 15 & 14 \\
$\mathrm{CN}^{-}(\mathrm{mM})$ & 0.07 & 0.1 & 0.03 \\
$\mathrm{OCN}^{-}(\mathrm{mM})$ & 0.12 & 0.09 & 0.02 \\
$\mathrm{NO}_{2}^{-}(\mathrm{mM})$ & 0.20 & 0.03 & 0.03 \\
$\mathrm{NO}_{3}{ }^{-}(\mathrm{mM})$ & 0.14 & 0 & 0 \\
$\mathrm{SO}_{4}{ }^{2-}(\mathrm{mM})$ & 0.63 & 0.15 & 0.23 \\
$\mathrm{NH}_{4}{ }^{+}(\mathrm{mM})$ & 0.03 & 0 & 0 \\
$\mathrm{Relación} \mathrm{molar} \mathrm{SO}_{4}{ }^{2-} / \mathrm{SCN}$ & 0.96 & 0.58 & 0.89 \\
$\mathrm{Relación} \mathrm{molar}_{\text {N-intermedios/SCN }}$ & 0.85 & 0.85 & 0.68 \\
\hline
\end{tabular}

*Concentración variable y relación másica $\mathrm{KMnO}_{4} / \mathrm{CN}$ de 3:1; $\mathrm{pH}=12$ y 20 min de agitación en el caso de la disolución con concentración inicial de $200 \mathrm{mg} / \mathrm{L}$, que también muestra una eliminación de tiocianatos ligeramente superior.

Finalmente, se observan diferencias en el balance molar $\mathrm{SO}_{4}{ }^{2-} / \mathrm{SCN}$ degradado (cuadro II) para las distintas disoluciones de tiocianato. En el caso de la concentración de $200 \mathrm{mg} / \mathrm{L}$, este balance molar es cercano a 1, lo que indica que todo el azufre de las moléculas de tiocianatos degradadas se oxidó a sulfato. Para concentraciones menores de tiocianato se obtuvieron balances molares de 0.5 a 0.9 , lo que sugiere la presencia de especies de azufre no detectadas en este estudio tales como sulfuros o polisulfuros (Collado et al. 2009, Viña et al. 2019). El balance de intermedios nitrogenados (relación molar $\mathrm{N}$-intermedios/SCN en el cuadro II) es ligeramente inferior a la unidad en todos los casos, sugiriendo la presencia de otras especies nitrogenadas además de las formas oxidadas nitrato, nitrito, amonio.

\section{Aguas residuales mineras}

Se estudiaron cuatro tipos de aguas residuales con distintas concentraciones de cianuro y tiocianatos, procedentes de diferentes áreas de una instalación minera local. Para una mejor compresión de los resultados, debe tenerse en cuenta que las prácticas habituales en instalaciones de la minería aurífera no incluyen el seguimiento de la concentración de tiocianatos en aguas residuales, ya que se trata de un contaminante no legislado.

Las figuras 3, 4 y 5 presentan la eliminación de cianuros de las aguas CIL, AP, APT y AD de la balsa de contención. En el caso del agua CIL (concentración inicial de cianuros de $312 \mathrm{mg} / \mathrm{L}$ y de tiocianatos de $314 \mathrm{mg} / \mathrm{L}$ ), se alcanzaron valores de eliminación de cianuros cercanos al $100 \%$ para la relación másica $\mathrm{KMnO} 4: \mathrm{CN}$ de 3:1. En el caso del agua de proceso (concentración residual de cianuros de $15 \mathrm{mg} / \mathrm{L}$ ) se obtuvieron porcentajes de eliminación crecientes con la relación másica $\mathrm{KMnO}_{4}: \mathrm{CN}$, si bien la degradación no fue completa para ninguna de las condiciones experimentales utilizadas. De manera análoga, en la figura 5 se observa que para el agua de drenaje de la bolsa de contención (concentración residual de cianuro de $1.9 \mathrm{mg} / \mathrm{L}$ ) se alcanzan valores de eliminación cercanos al $70 \%$. De manera general se observa que el porcentaje de eliminación de cianuros en las distintas aguas analizadas varió de la siguiente manera: agua CIL $>$ agua de proceso $>$ agua de drenaje. Es decir, el rendimiento de oxidación de cianuros aumenta a medida que aumenta la concentración inicial de cianuro en las aguas industriales. 


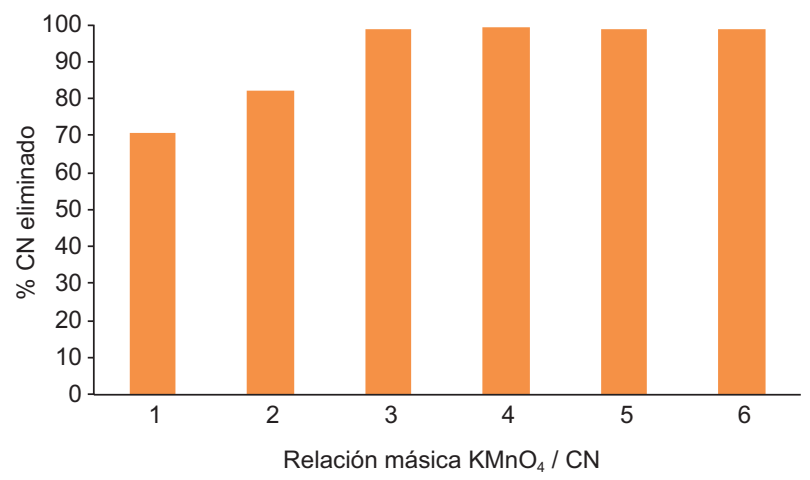

Fig. 3. Eliminación de cianuro $(\mathrm{CN})$ del agua residual CIL (concentración inicial de cianuro de $312 \mathrm{mg} / \mathrm{L}$ ) en función de la relación másica $\mathrm{KMnO}_{4} / \mathrm{CN}$.

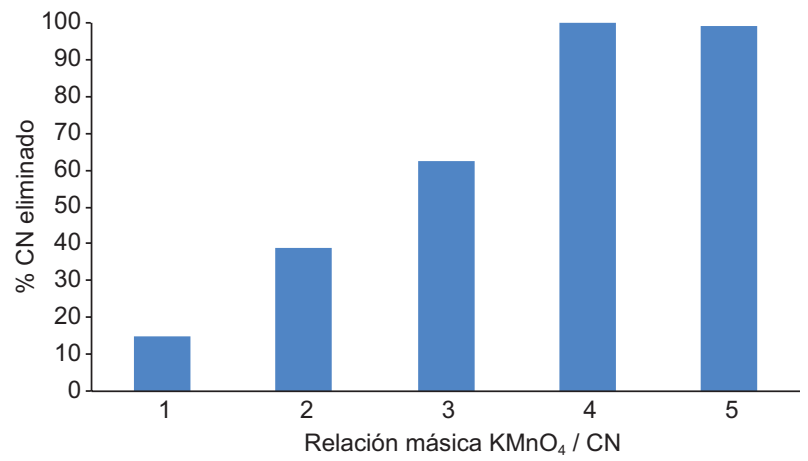

Fig. 4. Eliminación de cianuro $(\mathrm{CN})$ de agua de proceso pretratada con $\mathrm{SO}_{2}$ (concentración inicial de cianuro de 15 $\mathrm{mg} / \mathrm{L}$ ) en función de la relación másica $\mathrm{KMnO}_{4} / \mathrm{CN}$.

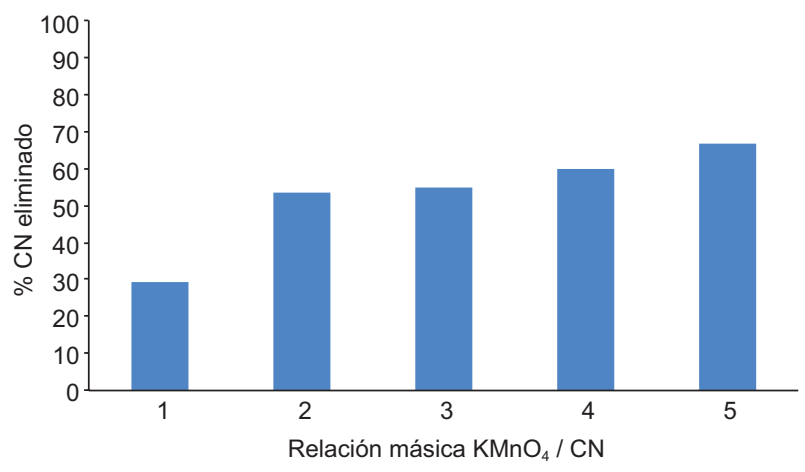

Fig. 5. Eliminación de cianuro $(\mathrm{CN})$ del agua de drenaje (concentración inicial de cianuro de $1.9 \mathrm{mg} / \mathrm{L}$ ) en función de la relación másica $\mathrm{KMnO}_{4} / \mathrm{CN}$.

Este comportamiento se debe a la oxidación parcial de los tiocianatos de manera simultánea durante la oxidación, los cuales liberan cianuro en la disolución tal y como se ha observado en el análisis de disoluciones sintéticas (Fig. 2b y Cuadro II). La degradación de tiocianatos es un proceso con bajos porcentaje de eliminación (Fig. 2a), por lo que su contribución es notable sólo cuando la concentración de cianuros en el agua a tratar es suficientemente baja (e.g., agua de drenaje y agua de proceso) como para que la cantidad de cianuros liberados a consecuencia de la degradación de tiocianatos represente una fracción relevante en la concentración final.

Finalmente, se evaluó la idoneidad de la oxidación con permanganato para la degradación de agua de proceso generada en continuo en la instalación minera, recogiendo alícuotas de agua cada $30 \mathrm{~min}$ durante $7 \mathrm{~h}$. Dichas muestras de agua se trataron con permanganato potásico con una relación másica $\mathrm{KMnO}_{4}: \mathrm{CN}$ de 5:1 (Fig. 6). Las diferentes alícuotas tomadas del agua de proceso presentaron concentraciones iniciales de cianuro de 33 a $50 \mathrm{mg} / \mathrm{L}$. En todos los casos se lograron porcentajes elevados de eliminación de cianuro cercanos al $95 \%$, si bien no se logró la degradación completa. Nuevamente, este comportamiento se debe a la liberación de cianuros por la degradación simultánea e incompleta de los tiocianatos por reacción con el permanganato.

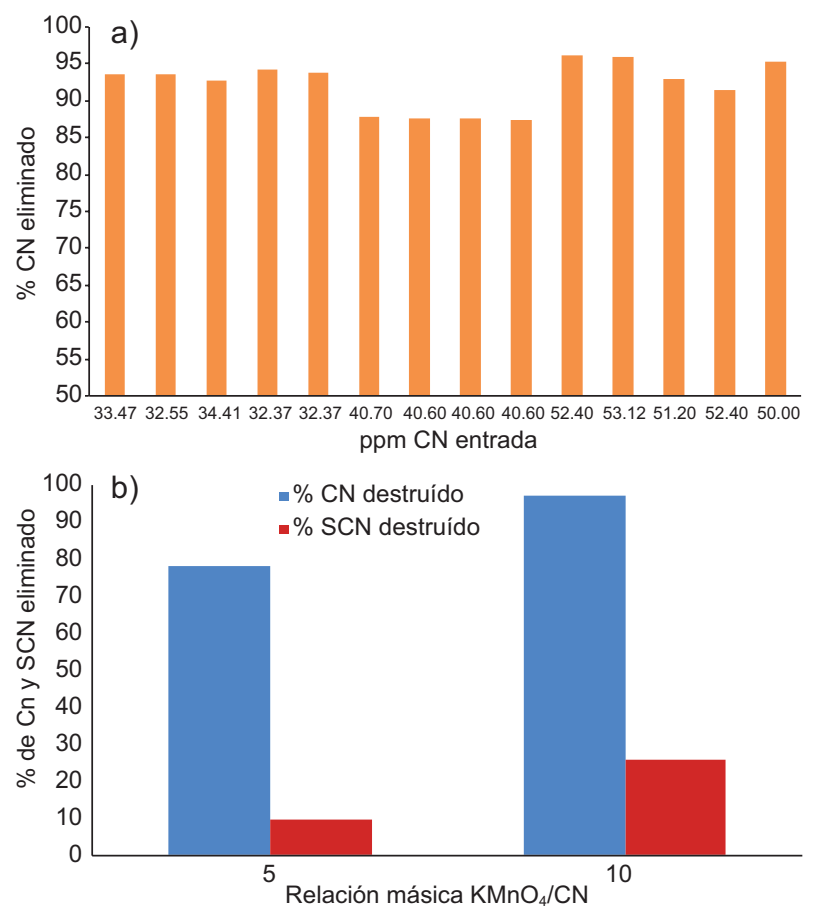

Fig. 6. (a) Eliminación de cianuro en agua de proceso recogida en continuo para una relación másica $\mathrm{KMnO}_{4} / \mathrm{CN}$ de 5:1; $\mathrm{pH}=12$ y 20 min de agitación. (b) Eliminación de cianuro y tiocianato en el agua de proceso recogida en continuo para relaciones másicas $\mathrm{KMnO}_{4} / \mathrm{CN}$ de 5 y 10 , $\mathrm{pH}=12$ y $20 \mathrm{~min}$. de agitación. 


\section{CONCLUSIONES}

El estudio realizado demostró que las propiedades oxidativas del permanganato de potasio son útiles para el tratamiento de aguas residuales que contienen cianuros y tiocianatos procedentes del sector de la minería aurífera. Se probó la capacidad del permanganato potásico para transformar ambas especies cianuradas en compuestos menos tóxicos, como cianatos, nitratos, amonio y nitritos, a partir de aguas sintéticas e industriales mineras.

Se evaluó la eliminación para diversas relaciones másicas permanganato:especie cianurada entre 1-7. En el caso de la oxidación de cianuros, la reacción es cuantitativa en presencia de exceso de permanganato y se alcanzan tasas de degradación cercanas al $100 \%$, detectándose nitratos como intermedios mayoritarios en disolución. La oxidación de tiocianatos es más difícil y menos cuantitativa, tal como se deduce de los resultados de oxidación de aguas sintéticas con similares relaciones másicas de oxidante. Se confirma además la liberación de cianuros en disolución durante la degradación de tiocianatos con permanganato potásico.

Los ensayos realizados con aguas residuales de mina muestran las mayores tasas de degradación de cianuros para las aguas residuales con mayor concentración inicial de cianuros. Este comportamiento se debe a la degradación simultánea de tiocianatos y la consiguiente liberación de cianuros en el medio, cuya contribución es notable cuando la concentración de cianuros en el agua a tratar es suficientemente baja.

Los datos sugieren que la degradación completa de especies cianuradas en aguas de minería empleando permanganato potásico como oxidante depende en gran medida del contenido de tiocianatos como resultado de su menor reactividad. Para alcanzar la depuración total de aguas residuales se precisaría su empleo en combinación con otros procesos.

\section{AGRADECIMIENTOS}

Agradecemos al Gobierno español por su apoyo a través de los programas MINECO (Ministerio de Economía y Empresa de España), becas CTM201456770-R y MAT2016-78155-C2-1-R.

\section{REFERENCIAS}

Ballester A., Verdeja L.F. y Sancho J. (2003). Metalurgia extractiva. Vol. I. Fundamentos. Síntesis, Madrid, $507 \mathrm{pp}$.
Botz M.M. y Terry T.I. (2002). Treatment of solutions and slurries for cyanide removal. En: Mineral processing plant: Design, practice, and control proceedings. Vol. 2. (Mular A.L., Halbe D.N. y Barratt D.J., Eds.). Society for Mining, Metallurgy and Exploration, Littleton, Colorado, 1866-1885.

Botz M.M., Mudder T.I. y Akcil A.U. (2005) Cyanide treatment: Physical, chemical, and biological processes. Develop. Miner. Process. 2005 (15), 672-702. https:// doi.org/10.1016/S0167-4528(05)15028-5

Botz M.M., Mudder T.I. y Akcil A.U. (2016). Cyanide treatment: Physical, chemical, and biological processes. En: Gold ore processing. 2a ed. (Adams M.D., Ed.). Elsevier, 619-645.

Bonan A., Teixeira L.A.C. y Kohler H.M. (1994). Kinetics of the oxidation of free and copper cyanides aqueous solutions with hydrogen peroxide En: Extraction and processing for the treatment and minimization of wastes (Hager J.P., Ed.). The Minerals, Metals and Materials Society, Warrendale, Pennsylvania, pp. 357-368.

Brady T. (2017) Thoughts on global gold mine supply. Gold Investor [en línea]. https://www.gold.org/ goldhub/research/gold-investor/gold-investor-september-2017/thoughts-on-global-gold-mine-supply $10 / 10 / 2018$

Budaev S.L., Aseev D.G., Khandarkhaeva M.S. y Batoeva A.A. (2014). Removal of thiocyanates by persulfates from gold mine wastewater. Proceedings of the IWA 6th Eastern European Young Water Professionals Conference "EAST Meets WEST". Istanbul. May 28-30, 28-30.

Chung J. y Wood J.L. (1970). Oxidation of thiocyanate to cyanide and sulfate by the lactoperoxidase-hydrogen peroxide system. Arch. Biochem. Biophys. 141, 73-78. https://doi.org/10.1016/0003-9861(70)90108-6

Collado S., Laca A. y Díaz, M. (2009). Wet oxidation of thiocyanate under different $\mathrm{pH}$ conditions: Kinetics and mechanistic analysis. Ind. Eng. Chem. Res. 48, 9902-9909. https://doi.org/10.1021/ie9006485

Collado S., Laca A. y Díaz M. (2010). Catalytic wet oxidation of thiocyanate with homogeneous copper (II) sulphate catalyst. J. Hazard. Mater. 177, 183-189. https://doi.org/10.1016/j.jhazmat.2009.12.015

Dabrowski B., Zaleska A., Janczarek M., Hupka J. y Miller J.D. (2002). Photo-oxidation of dissolved cyanide using $\mathrm{TiO}_{2}$ catalyst. Chemistry J. Photochem. Photobiol. A Chem. 151, 201-205. https://doi.org/10.1016/s10106030(02)00151-x

Dash R.R., Gaur A. y Balomajumder C. (2009). Removal of cyanide from water and wastewater using granular activated carbon. J. Hazard. Mater. 163, 1-11. https://10.1016/j.cej.2008.06.021 
Degrémont S.A.E. (1991). Water treatment handbook. 6a ed. Springer-Verlag, $1596 \mathrm{pp}$.

Devuyst E.A., Conard B.R. y Ettel V.A. (1982). Pilot plant operation of the Inco $\mathrm{SO}_{2} /$ air cyanide removal process. Can. Min. J. 8, 27-30.

Douglas W., King M., Mohapatra B., Cameron R., Kapoor A. y Koren D. (2012). A critical review on destruction of thiocyanate in mining effluents. Miner. Eng. 34, 38-47. https://doi.org/10.1016/j.mineng.2012.04.009

Elsner L. (1846). Observations on the behavior of pure metals in an aqueous solution of cyanide (in German). J. Praktische Chemie 37, 441-446.

Fernández B. (2007). Desarrollo de un nuevo método para la eliminación de cianuro de aguas residuales de mina. Tesis Doctoral. Escuela de Minas, Universidad de Oviedo, España, 201 pp. [en línea]. http://hdl.handle. net/10803/31849

Kuyucaka N. y Akcil A. (2013). Cyanide and removal options from effluents in gold mining and metallurgical processes. Miner. Eng. 50-51, 13-29. https://doi. org/10.1016/j.mineng.2013.05.027

Logsdon M.J., Hagelstein K. y Mudder T.I. (1999). The management of cyanide. Gold Extraction International Council on Metals and the Environment, Ottawa, Ontario, $10 \mathrm{pp}$.

Logsdon M.J., Hagelstein K. y Mudder T.I. (2004). El manejo del cianuro en la extracción de oro. Trad. de The management of cyanide in gold extraction, Consejo Internacional de Metales y Medio Ambiente (ICME).

Marecaux E.N. (2006). Effects of potassium permanganate on the sailfin molly, Poecilia latippinna, at varying salinity levels. M.Sc thesis. University of Florida, Gainesville, Florida, 55 pp.

Medialdea J.M., Arnaiz C. y Díaz E. (2005). El permanganato potásico $\left(\mathrm{KMnO}_{4}\right)$ en el tratamiento de aguas residuales. Tecnología del Agua 25 (264), 56-66.

Medina S., Torres M., Durán Y., Ramírez R., Herrera J. y Ramírez P. (2012). The thiocyanate degradation by fungi isolated from mining environments and evaluation of degradative capacity. Revista Peruana de Biología 19 (1), 081-088.

Mekuto L., Ntwampe S.K.O., Utomi C.E., Mobo M., Mudumbi J.B., Ngongang M.M. y Akinpelu E.A. (2017). Performance of a continuously stirred tank bioreactor system connected in series for the biodegradation of thiocyanate and free cyanide. J. Environ. Chem. Eng. 5, 1936-1945. https://doi.org/10.1016/j.cej.2009.06.041

Nagashima S. y Ozawa T. (1981). Spectrophotometric determination of cyanide with isonicotinic acid and barbituric acid. Int. J. Environ. Anal. Chem. 10, 99-106. https://doi.org/10.1080/03067318108071535

Oulego P., Collado S., Laca A. y Diaz M. (2014) Simultaneous oxidation of cyanide and thiocyanate at high pressure and temperature. J. Hazard. Mater. 280, 570578. https://doi.org/10.1016/j.jhazmat.2014.08.051

OMS 2003. Cyanide in drinking-water. Documento de referencia para la elaboración de las Guías de la OMS para la calidad del agua potable. Organización Mundial de la Salud, Ginebra, Suiza.

Ordiales M., Fernández D., Verdeja L.F. y Sancho J. (2015). Potassium permanganate as an alternative for gold mining wastewater treatment. JOM-J. Min. Met. Mat. S. 67 (9), 1975-1985. https://doi.org/10.1007/s11837-015-1547-9

Pizzi N.G. (2010). Water treatment: Principles and practices of water supply operations. American Water Works Association, Denver, Colorado, 560 pp.

Rancaño Pérez A. (2012). Degradación biológica de tiocianato. Tecnología del Agua 32 (36), 66-76.

Sancho J.P., Fernández B., Ayala J., García M.P. y Lavandeira A. (2009). Uses of the potassium permanganate to eliminate copper cyanide from wastewater resulting from a lixiviation plant in a gold mine (I). Rev. Metal. 45 (415). https://doi.org/10.3989/revmetalm.0846

Soto H., Nava F., Leal J. y Jara J. (1995). Regeneration of cyanide by ozone oxidation of thiocyanate in cyanidation tailings. Miner. Eng. 8, 273-281. https://doi. org/10.1016/0892-6875(94)00123-T

SSA 1994. Norma Oficial Mexicana NOM-127-SSA1-1994. Salud ambiental, agua para uso y consumo humano. Límites permisibles de calidad y tratamientos a que debe someterse el agua para su potabilización. Secretaría de Salud. Diario Oficial de la Federación, México, 18 de enero.

UNE 2009. UNE-EN ISO 10304-1:2009. Calidad del agua. Determinación de aniones disueltos por cromatografía de iones en fase líquida. Parte 1. Determinación de bromuro, cloruro, fluoruro, nitrato, nitrito, fosfato y sulfato. 11 de noviembre.

UNE 2012. UNE-EN ISO 14403-1:2012 Calidad del agua. Determinación del cianuro total y del cianuro libre por análisis en flujo (FIA y CFA). Parte 1: Método por análisis de inyección en flujo (FIA). 26 de diciembre.

Vella P. (1996). Sixth International Symposium on Chemical Oxidation, Vanderbilt University, Nashville, Tennessee. Technomic, 28-30.

Vicente J. y Díaz M. (2003). Thiocyanate wet oxidation. Environ Sci. Tech. 37, 1452-1456. https://doi. org/10.1021/es020103c

Viña Mediavilla J.J., Fernández-Pérez B., Fernández de Córdoba M.C., Ayala Espina J. y Ania C.O. (2019). Photochemical degradation of cyanides and thiocyanates from an industrial wastewater. Molecules 24 (7), 1373-1387. https://doi.org/10.3390/molecules24071373

Yarar B. (2002). Long term persistence of cyanide species in mine waste environments En: Proceedings of the 9th International Conference on Tailings and Mine Waste CRC Press, p. 201. 